\title{
Towards Crystallographic Orientation and Strain Mapping of 1D \& 2D Tellurium from 4D-STEM
}

Alejandra Londono-Calderon ${ }^{1}$, Benjamin Savitzky ${ }^{2}$, Darrick Williams ${ }^{1}$ and Michael Pettes ${ }^{1}$

${ }^{1}$ Center for Integrated Nanotechnologies (CINT), Los Alamos National Laboratory, Los Alamos, New Mexico, United States, ${ }^{2}$ National Center for Electron Microscopy (NCEM), Molecular Foundry, Lawrence Berkeley National Laboratory, Berkeley, California, United States

Tellurium nanostructures are attractive candidates for future electronic and functional materials due to their narrow band-gap and tunable electronic and optical properties [1,2]). The anisotropic helical crystal structure of trigonal tellurium (t-Te) and the weak van der Waals stacking of its layers; allows the formation of a variety of 1D nanostructures such as nanowires and nanobelts. A kinematically driven growth can promote the formation of 2D tellurium known as tellurene. Standard HRTEM images provide localized crystallographic information, which usually leaves out larger-scale structural components. Longrange strain fields, caused by lattice misorientation, defects, or anisotropic growth, is often not fully captured in this type of analysis. 4D-STEM provides a remarkable tool for structural analysis across many length scales, and enables extracting the crystalline structure, orientation, virtual images, and strain maps of a wide variety of nanomaterials [3].

In this work, we have synthesized 1D and 2D tellurium nanostructures using a microwave-assisted solution chemistry method and identified crystallographic variations and strain maps using 4D-STEM datasets. Fast collection of thousands of NBED patterns of beam sensitive Te nanowires and flakes was performed using a Gatan K3 direct electron detector, and these data sets were used to identify variations in the crystallographic orientation along the axis of the 1D Te wires and 2D Te flakes with grain boundaries. Classification and segmentation of common diffraction patterns was performed using the open software py4DSTEM [4] and are shown in Figure 1 for a single ultrathin Te nanowire. For the 2D tellurene flakes, the preferential growth along the [-12-10] direction (perpendicular to the helical chain) allows the formation of grain boundaries within the structure. Figure 2 shows 4D-STEM data near the grain boundary where the area is classified in two classes (top and bottom grains) with the dame orientation and rotated $75^{\circ}$. Strain mapping on both regions was obtained by evaluating the change in lattice vector in the dataset. 


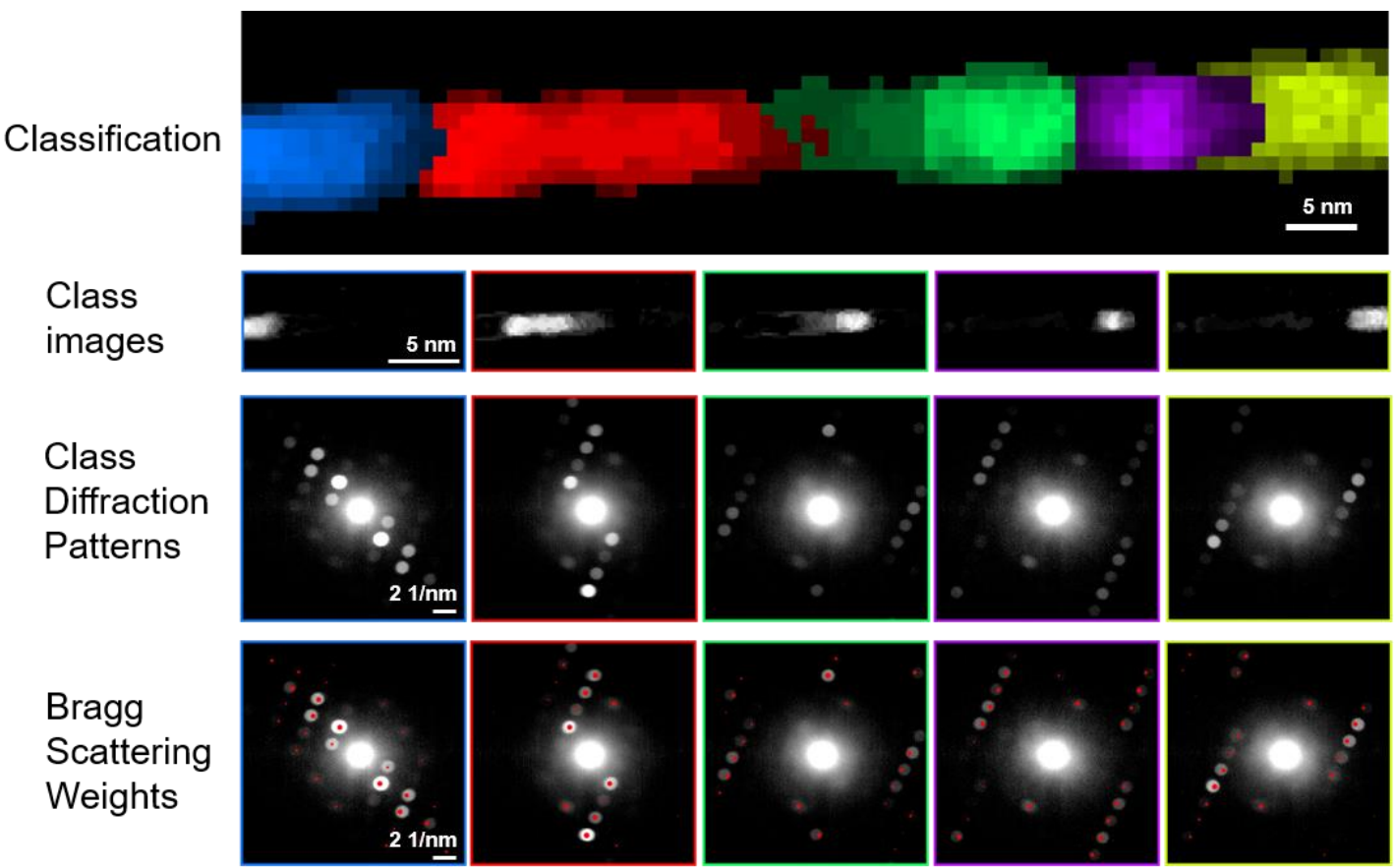

Figure 1. 4D-STEM classification of crystallographic orientations of a single t-Te nanowire. Each color in the classification image represents a region with a distinct crystallographic orientation, while the corresponding class diffraction patterns are the weighted average NBED pattern over the scan positions. The final row shows the relative weights associated with various directions of Bragg scattering for each class
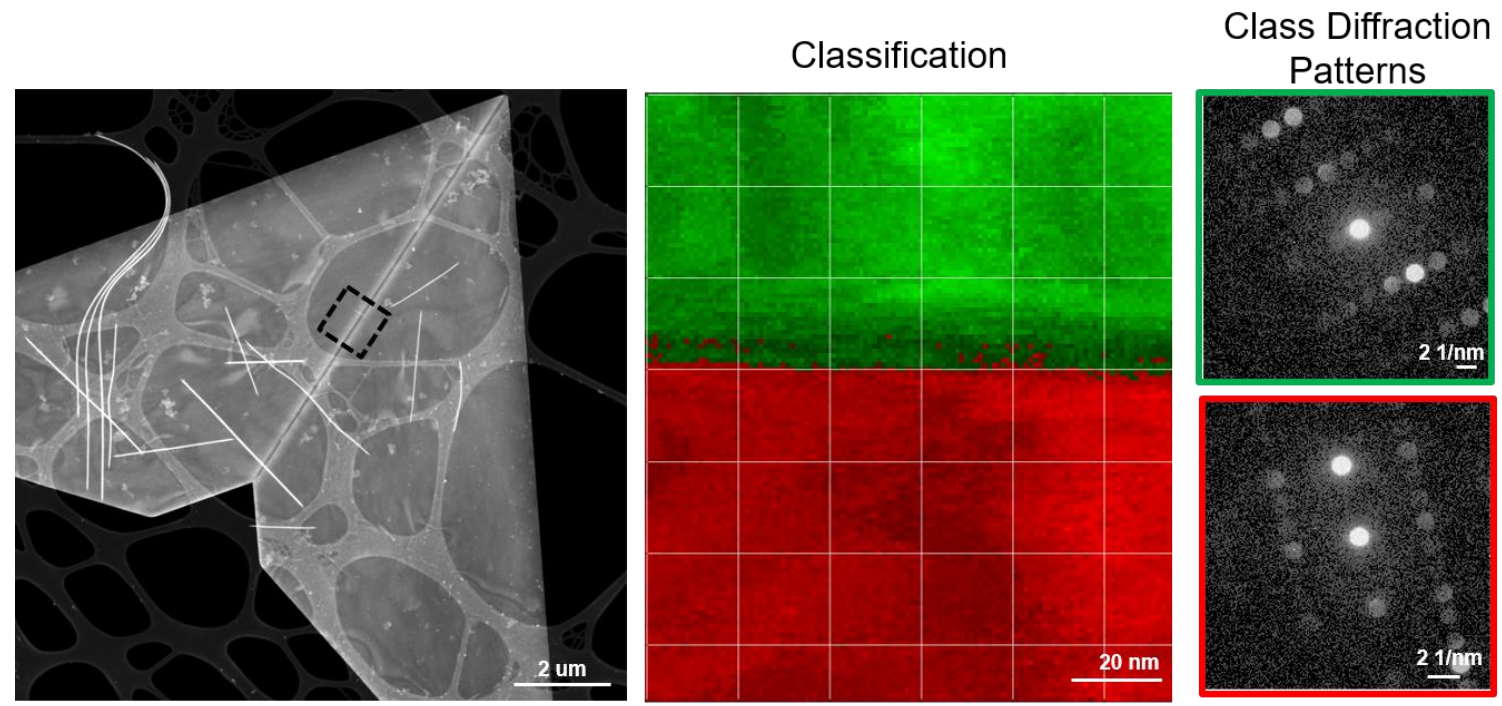

Figure 2. Analysis of a grain boundary in $2 \mathrm{D}$ tellurene. Near the interface, the crystallographic orientation is the same but rotated

\section{References}

[1] W. Wu, G. Qiu, Y. Wang, R. Wang, and P. Ye. Chem. Soc. Rev. 47, 7203 (2018).

[2] M. Amani, C. Tan, G. Zhang, C. Zhao, J. Bullock, X. Song, H. Kim, V. R. Shrestha, Y. Gao, K. B. Crozier, M. Scott, and A. Javey. ACS Nano 12, 7253 (2018).

[3] C. Ophus. Microscopy and Microanalysis, 25, 563 (2019).

[4] https://github.com/py4dstem/py4DSTEM 